BNL-107821-2015-JA

\title{
Laser ion source activities at Brookhaven National Laboratory
}

\author{
Masahiro Okamura and Takeshi Kanesue
}

Submitted to Radiation Effects and Defects in Solids

April 2015

Collider-Accelerator Department

Brookhaven National Laboratory

\section{U.S. Department of Energy \\ Office of Science, Office of Nuclear Physics}

Notice: This manuscript has been authored by employees of Brookhaven Science Associates, LLC under Contract No. DE-SC0012704 with the U.S. Department of Energy. The publisher by accepting the manuscript for publication acknowledges that the United States Government retains a non-exclusive, paid-up, irrevocable, world-wide license to publish or reproduce the published form of this manuscript, or allow others to do so, for United States Government purposes. 


\section{DISCLAIMER}

This report was prepared as an account of work sponsored by an agency of the United States Government. Neither the United States Government nor any agency thereof, nor any of their employees, nor any of their contractors, subcontractors, or their employees, makes any warranty, express or implied, or assumes any legal liability or responsibility for the accuracy, completeness, or any third party's use or the results of such use of any information, apparatus, product, or process disclosed, or represents that its use would not infringe privately owned rights. Reference herein to any specific commercial product, process, or service by trade name, trademark, manufacturer, or otherwise, does not necessarily constitute or imply its endorsement, recommendation, or favoring by the United States Government or any agency thereof or its contractors or subcontractors. The views and opinions of authors expressed herein do not necessarily state or reflect those of the United States Government or any agency thereof. 
Laser Ion Source activities at Brookhaven National Laboratory

Takeshi Kanesue

Brookhaven National Laboratory, Collider-Accelerator Department, Upton, New York 11973, USA

Phone: +1-631-344-4777

Mail: tkanesue@,bnl.gov

Masahiro Okamura

Brookhaven National Laboratory, Collider-Accelerator Department, Upton, New York 11973, USA

Phone: +1-631-344-3577

Mail: okamura@bnl.gov 
Abstract

In Brookhaven National Laboratory (BNL), we have been developing laser ion sources for diverse accelerators. Tabletop Nd:YAG lasers with up to several Joules of energy are mainly used to create ablation plasmas for stable operations. The obtained charge states depend on laser power density and target species. Two types of ion extraction schemes, Direct Plasma Injection Scheme (DPIS) and conventional static extraction, are used depending on application. We optimized and select a suitable laser irradiation condition and a beam extraction scheme to meet the requirement of the following accelerator system. We have demonstrated to accelerate more than $5 \times 10^{10}$ of $\mathrm{C}^{6+}$ ions using the DPIS. We successfully commissioned low charge ion beam provider to the user facilities in BNL. To achieve higher current, higher charge state and lower emittance, further studies will continue.

Key words: Laser ablation; Laser ion source; Particle accelerator;

\section{Introduction}

In BNL since 2006, we have studied how to provide a high brightness heavy ion beams from laser ablation plasmas to diverse accelerators. Currently we have several projects of laser ion sources (LIS) going on simultaneously and some of them have already showed fruitful results. Here, we like to introduce what we have achieved and what are needed to be solved in our projects. Let us note that there are some types of LIS in the field. For instance, some are using resonances with laser wavelengths and ionization energies to obtain particular charged particles. Others attempt to induce high-energy ions from laser irradiations of thin foil targets. Among them, our interest is to irradiate laser beam on a thick solid target and heat the induced plasma with classical laser energy absorption process to provide ion beams to radio frequency driven accelerators. Probably this type of LIS has the longest history in the field since the idea is extremely simple. In the following part of the article, we only discuss on this type of LIS.

\section{Advantages of LIS}

We believe that a LIS has a couple of fundamental advantages comparing to other ion sources. The first is a size of the plasma while ionization occurs. Other ion sources have a certain size of plasma volume and are much larger than the initial laser plasma. The extracted ions form a beam and a density distribution of ions is always reflected from an image of the initial plasma shape behind the extraction electrodes. A LIS has a very small plasma volume and it can be assumed as a pinpoint image source. This enables us to form a clean and uniform beam that can eliminate nonlinearity of the space charge force in the beam. Next, the plasma temperature can be suppressed at the ion extraction. The laser irradiation on the target makes high-density plasma and it expands adiabatically while moving away from the target. At the extraction point, the plasma is already cooled. In other words, the fraction of the plasma captured by an ion extraction hole comparing to the entire expanding plasma is very small so that the extracted ion beam has a very limited thermal distribution. So a LIS has intrinsic advantages to provide high brightness beams. In our developments, we have always tried to urge these features.

\section{Activities in BNL}

Currently we are using three types of Nd:YAG laser systems, Thales SAGA 230 (1064 nm, $2.3 \mathrm{~J}$, $6 \mathrm{~ns})$, Ekspla SL334 (1064 nm, $500 \mathrm{~mJ}, 170$ - $500 \mathrm{ps})$ and Qunatel Brilliant B (850 mJ $1064 \mathrm{~nm}, 6 \mathrm{~ns})$. All three lasers are tabletop type and commercially available models. We used to have a $\mathrm{CO}_{2}$ laser, however it was not easy to obtain stable ion beams using the laser and achieved laser power density was not high enough due to relatively longer pulse length. So we stopped to use it. To achieve the highest charge state by using the Nd:YAG lasers, we normally focus laser beam as much as possible. To obtain lower charge state ions, the laser spot size is adjusted to maximize a desired charge state. We also select ion extraction methods to meet the application's demands. For moderate ion beam current production, we use conventional static electric field extraction, which suits for less space charge effect condition. To obtain space charge dominant beam, we apply direct plasma injection scheme (DPIS). The DPIS was invented to preserve high brightness of laser ablation plasma until the ions are trapped by radio frequency acceleration buckets. We believe this is the best scheme for very high current heavy ion acceleration (1). In the following, we will introduce our projects one by one. 


\subsection{Challenge of high current high charge state heavy ion beam production}

We started the DPIS experiment using $\mathrm{C}^{4+}$ and $\mathrm{C}^{6+}$ since carbon can be ionized up to fully stripped relatively easily. The accelerated peak current detected after RF acceleration reached $35.8 \mathrm{~mA}$ and $32.8 \mathrm{~mA}$, and the accelerated particle numbers from single laser shot were counted as $1.2 \times 10^{11}$ and $5.2 \times 10^{10}$ particles per pulse for $\mathrm{C}^{4+}$ and $\mathrm{C}^{6+}$ respectively (2).

The typical experimental set up is shown in Fig. 1. A target is placed in a high voltage region and hit by a laser beam. The induced plasma expands and moves away from the target. The moving velocity of the gravity center of the plasma has a correlation to the initial plasma temperature. The plasma expanding angle is about 20 degrees from its axis. Then only the center part of plasma enters into solenoid magnet which can suppress the expanding angle of the plasma slightly in the magnet. This suppression of the expanding angle by the longitudinal field is small. However the effect on the total ion beam current is quite large. Only a few hundred gauss enhances the current more than ten times (3). The field strength of the magnet is adjusted not to exceed a space charge limit at the extraction point.

The plasma moves through the magnet and enters into the following radio frequency quadrupole (RFQ) accelerator. This means the high brightness low temperature ions are delivered within neutral plasma state and the degradation caused by nonlinear space charge force can be eliminated. Up to this extraction point in the RFQ, the moving path of the plasma is completely surrounded by metal walls even in the solenoid magnet. So plasma expands freely at the constant potential in longitudinal direction until the plasma reaches the exaction point. A high voltage potential is applied to the isolated volume including plasma target and expanding space. The entire electrically isolated structure is surrounded by a vacuum vessel and the solenoid pipe, those are grounded. So we don't need to build high voltage fences around the ion source. Even during the operation, we can work on the laser beam path. In the RFQ, the plasma extraction hole is at the end wall of the RFQ's resonating cavity. The extraction hole size is almost comparable to the bore size of RFQ channel, which is typically several $\mathrm{mm}$ in diameter. The applied voltage to the isolated volume corresponds to the designed injection velocity of the RFQ. The extraction voltage gap is created between the extraction hole and the RFQ's electrodes. Of course, the potentials of each electrode oscillate at RF frequency which is at $100 \mathrm{MHz}$ in our case. So that the extraction hole has a static positive voltage and the electrodes make quadrupole field which has $10 \mathrm{~ns}$ of sinusoidal period. This is an enough time to kick out electrons efficiently but ions can be captured by the RFQ field. At the plasma sheath, the ion beam current is limited by a space charge limit, however the plasma has in initial moving velocity and this can increase the limit.

On the other hand, we need to pay attention to the one of the difficulties at the ion extraction of the DPIS. We have a magnetic field leakage from the confinement solenoid, static electric field caused by the positive voltage fed to the extraction hole and RF focusing electric field by the RFQ electrodes simultaneously at the same volume where the plasma extraction sheath is created. It is not a simple issue to understand the behavior of the plasma and high-density ion beam at the extraction in the DPIS. Also the weak magnetic field leakages easily trigger breakdowns since the emitted electrons from the positive extraction surface follow the direction of the magnetic field. These are the main issues we are trying to solve to obtain brighter ion beams using DPIS.

To create highly charged ions, a high plasma temperature is required $(4,5)$. To achieve the high temperature, an efficient energy deposition on the plasma by laser photons is important. Before the plasma spreads, it should be abruptly heated by a laser irradiation. Using the laser systems we have, probably up to $10^{13} \mathrm{~W} / \mathrm{cm}^{2}$ of laser power density is achieved and we may neglect nonlinear or multi photon absorption process and the heating energy is mostly given by inverse bremsstrahlung. As long as a thermal equilibrium can be reached, a shorter laser pulse length is better. Using Thales SAGA 230 (2.3 J, $6 \mathrm{~ns})$, we can produce $\mathrm{Al}^{11+}, \mathrm{Fe}^{20+}$ and $\mathrm{Ag}^{20+}(6)$. However, we could not obtain highly charged ions from heavier elements like $\mathrm{Au}$ or Ta. To create higher charge state, recently, we started to use Ekspla SL344 which has sub nanosecond pulse width. This laser enables us to see $10^{14} \mathrm{~W} / \mathrm{cm}^{2}$ which is one order greater value served by the SAGA 230. We are currently exploring the laser irradiation condition, however the pulse length seems too short to have an equilibrium of plasma heating since obtained plasma consists of high and low velocity components. Some more efforts and new strategies are being considered.

\subsection{Intense low charge state very heavy ion beam production}

We have proposed to use DPIS for heavy-ion inertial confinement fusion (HIF) (7). DPIS may help to mitigate strong space charge force at a low energy beam transport line which is between an ion 
source and the first front end accelerator in the HIF application. In HIF scenarios, single or double charge state ions are assumed, since the lower charge state with heavy mass ions reduce space charge force especially in final focusing on the fusion target $(8,9)$. We assume $1 \mathrm{MJ}$ of input energy on a DT target at 1 $\mathrm{GeV}$. The ion sources need to provide at least 1 milli coulomb in total. If we have two hundreds sets of injectors for an entire facility, each ion source is responsible to provide $100 \mathrm{~mA}$ and $50 \mu$ s singly charged ions or $200 \mathrm{~mA} 50 \mu \mathrm{s}$ for charge state $2+$ ions. In case of $2+$ ions, the required accelerator cavity length becomes half, but the current will be doubled to achieve the same particle number. To verify the feasibility of a HIF LIS, We have tested productions of Au and Bi beams. The obtained performances indicated to fulfill the proposed common requirements of the HIF by some extrapolations.

However, to utilize a HIF LIS, a very important issue is remained. The current time profile of a LIS follows a shifted Maxwell-Boltzmann distribution so that the obtained current has a single peak and after that it decays gradually. In the design of the HIS accelerator complexes, every component must have enough space charge limit. To use the design limit efficiently, a square waveform of the beam current is desired instead of the typical LIS waveform. So we need to tailor the beam current. To overcome this issue, we are proposing to use a pulsed solenoid magnet. As explained previously, we have used static solenoid field to suppress the diverging angle of the expanding plasma. This can increase entire beam current in a single ion beam pulse. The idea is to apply short pulsed magnetic field on only a part of the beam pulse. This technique may enable us to form a square shape beam current profile of a LIS in the future. Some preliminary tests have been carried out (10). We assembled $100 \mathrm{~mm}$ length 50 turn solenoid and placed about $300 \mathrm{~mm}$ from a laser target. The ramp rate is around $10 \mu$ s which is fast enough for the slow expanding low charge state ions. We observed current profile change as expected and measured beam emittance variation within a pulse. We confirmed that beam emittance was also affected by the changing density of ion flux enhanced by the pulsed solenoid. Our next step is to tailor the current profile more precisely. Also we plan to control the extraction electric field. We believe the combination of the pulsed solenoid and controlled extraction field guide us to realize a square shape current profile with constant minimized beam emittance.

3.3 Low charge LIS with conventional extraction for feeding electron beam ion source.

In BNL, we have two major heavy ion user facilities and we started to provide various heavy ion beams to them from a low charge state laser ion source named as LION since 2014 (11). The facilities are NASA space radiation laboratory (NSRL) and relativistic heavy ion collider (RHIC). The LION produces $\mathrm{C}^{+}, \mathrm{Al}^{+}, \mathrm{Si}^{+}, \mathrm{Ti}^{+}, \mathrm{Fe}^{+}, \mathrm{Ta}^{+}$, and $\mathrm{Au}^{+}$beams and those beams are delivered to electron beam ion source (EBIS) where ions are stripped up to required charge state (12). The highly ionized ion beams are extracted from the EBIS and then injected into the four rod type RFQ. The injection and extraction beam energies of the RFQ are $17 \mathrm{keV} / \mathrm{u}$ and $300 \mathrm{keV} / \mathrm{u}$ respectively. The inter-digital drift tube linear accelerator (IH-DTL) accelerates these ions to $2.0 \mathrm{MeV} / \mathrm{u}$ and sends them to booster synchrotron. Figure 4 shows a layout of the heavy ion injector. For NSRL operation, the beams of various ion species accelerated to desired energies by the booster synchrotron are supplied to the NSRL target room. The maximum beam energy of the facility is about $1.0 \mathrm{GeV} / \mathrm{u}$. In the NSRL, biological effects of high-energy cosmic rays are mainly investigated. Fast switching of ion species within 1 second is required to simulate cosmic rays precisely. External ion injection from LIS is ideal for this purpose. The number of target species is only limited by target holder dimension. Because pulsed plasma is generated from a solid state target and only ions are extracted and injected into an EBIS, there is no effect from preceding ion beam in principle. The booster synchrotron also has another extraction beam line connected to alternate gradient synchrotron (AGS) and the accelerated beams are finally delivered to the RHIC which is a double track collider ring consisting super conducting magnets. The circumference of the RHIC is $3.8 \mathrm{~km}$ and beam energy can be up to $100 \mathrm{GeV} / \mathrm{u}$.

Since the LION is routinely providing heavy ions to entire hadron facilities, a superior reliability is requested. Figure 5 shows a cross section of the LION. We employed a robust single Nd:YAG crystal laser (Quantel Brilliant B, $6 \mathrm{~ns} 850 \mathrm{~mJ}$ ) and an extra vacuum evacuation path at the beam extraction chamber to remove the exceeded plasmas and neutral vapors. The laser target holder is mounted on a two dimensional motorized stage and multiple different material plates are placed side by side in the holder. To switch the ion species, simply the motorized stage moves to provide desired material at the laser spot. The laser spot size is about $5 \mathrm{~mm}$ in diameter to keep the laser power density below $10^{9} \mathrm{~W} / \mathrm{cm}^{2}$ for singly charged ion production (13). For long time stable operation, target is scanned with very slow rate. For instance, the gold plate moves $0.1 \mathrm{~mm}$ for every 40 shots so that the entire surface is evenly consumed. As 
shown in Fig.5, the laser system is placed at the top of the frame and the laser beam aims the target with 30 degree of the incident angle. The induced plasma is guided by a $3.0 \mathrm{~m}$ long solenoid magnet with a several Gauss of the longitudinal magnetic field. The ion beam is extracted from $15 \mathrm{~mm}$ diameter aperture. The nominal beam emittance injected into the EBIS is about $0.06 \pi \mathrm{mm} \mathrm{mrad}$ (RMS) with up to $40 \mathrm{keV}$ of total beam energy. The maximum repetition rate is $5 \mathrm{~Hz}$. The LION can be operated for several months without major maintenance.

The successful operation of the LION indicated that a LIS can supply stable beams for a long period for major user facilities. Next, we plan to upgrade LION to achieve better injection emittance using a newly designed pulsed solenoid which was mentioned before.

3.4 Conventional extraction high charge state LIS.

BNL is developing high charge state LIS with conventional extraction system under the collaboration with KEK in Japan. At the moment, a new laser irradiation chamber fabricated by KEK is being tested in BNL (14). We successfully demonstrated to induce fully stripped carbon ions and various species are being examined to optimize the laser irradiation conditions. In the vacuum chamber, the laser path is fully separated by a stainless steel made enclosure. A $150 \mathrm{~mm}$ x $150 \mathrm{~mm}$ flat target can be installed to provide long time operation without breaking vacuum. We plan to install the LIS at an induction synchrotron called KEK digital accelerator (KED-DA) at Tsukuba campus of KEK next year.

\subsection{LIS for cancer therapy accelerator}

A next generation cancer therapy accelerator complex named as ion rapid cycling medical synchrotron (iRCMS) is being designed in BNL. The iRCMS consists of a DPIS-LIS, an RFQ, two IHDTLs, a high repetition rate synchrotron and a beam delivery system. The injector (the source and the linacs) of the iRCMS is required to provide both proton beams and pure fully stripped carbon beams at 8 $\mathrm{MeV} / \mathrm{u}$ to the main ring at a $15 \mathrm{~Hz}$ rep rate. Since the performance of the laser ion source has already been proved for high current carbon beam production, proton beam production was newly tested assuming the identical ion source set up for carbon beam generation. As a laser target for proton beam production, we have tested, metal hydrides and plastics. Generally metal hydride can provide proton beam efficiently though it is very difficult to form a plate shape since it is very fragile. Powder of some hydrides can be compressed to make a target relatively easily but it doesn't suit for long time operation. Plastic material contains hydrogen and it can be used as a target. However, most of plastics are transparent against fundamental wave length of Nd:YAG laser and ion beam production efficiency is not good. Now we are testing plastic plate coated by a thin metal layer which may encourage creating an initial plasma which leads efficient energy absorption. Some experimental results will be reported probably in next year.

\section{Conclusion}

In BNL, we have been studying laser ablation plasma and high brightness LIS for particle accelerator application with tabletop Nd:YAG lasers with up to several Joules of laser energy. Both high charge state and low charge state ion production of various species are investigated by controlling laser power density on a target. A LIS uses ions within only center part of adiabatically expanding plasma, so non-linearity of the space charge forces is minimized. Also extracted ion beam has a very limited thermal distribution. Therefore, a LIS has intrinsic advantages to provide high brightness beams. Two types of extraction methods are used at BNL depending on beam current to meet the requirements of a following accelerator system. For space charge dominant very high intensity beam, DPIS is used. For moderate beam current, ions are extracted by conventional static electric field. We demonstrated to accelerate $\mathrm{C} 4+$ and $\mathrm{C} 6+$ by RFQ with the detected peak current of more than $30 \mathrm{~mA}$. The DPIS was used for the experiments. Since 2014, a LIS, so called LION, started to provide $\mathrm{C}^{+}, \mathrm{Al}+, \mathrm{Si}+, \mathrm{Ti}^{+}, \mathrm{Fe}+, \mathrm{Ta}^{+}$, and $\mathrm{Au}+$ beams in combination with EBIS for NSRL and RHIC user operation. We proved that a LIS is able to supply stable beam for long-term user operation. Furthermore, a LIS for cancer therapy machine, a LIS for induction accelerator, a LIS for HIF driver are also being developed.

\section{Acknowledgement}

This work has been supported by Brookhaven Science Associates, LLC under Contract No. DEAC02-98CH10886 with the U.S. Department of Energy, and by the National Aeronautics and Space Administration 
5. References

(1) Okamura, M.; Takeuchi, T.; Jameson, R.A.; Kondrashev, S.; Kashiwagi, H.; Sakakibara, K.; Kanesue, T.; Tamura, J.; Hattori, T. Rev. Sci. Instrum. 2008, 79, $02 \mathrm{~B} 314$.

(2) Kanesue, T.; Okamura, M.; Kondo, K.; Tamura, J; Kashiwagi, H.; Zhang, Z. Rev. Sci. Instrum. 2010, 81, $02 \mathrm{~B} 723$.

(3) Okamura, M.; Adeyemi, A.; Kanesue, T.; Tamura, J.; Kondo, K. Rev. Sci. Instrum. 2010, 81, 02A510.

(4) Láska, L.; Jungwirth, K.; Krása, J.; Pfeifer, M.; Rohlena, K.; Ullschmied, J.; Badziak, J.; Parys, P.; Wolowski, J.; Gammino, S. et al, Appl. Phys. Lett. 2005, 86, 081502.

(5) Torrisi, L.; Gammino, S.; Picciotto, A.; Margarone, D.; Laska, L.; Krasa, J.; Rohlena, K.; Wolowski, J. Rev. Sci. Instrum. 2006, 77, $03 \mathrm{~B} 708$

(6) Okamura, M.; Kondrashev, S. Proceedings of Particle Accelerator Conference 07, Albuquerque, New Mexico, USA, 2007, p. 3761-3765.

(7) Masahiro, O.; Sekine, M.; Takahashi, K.; Kondo, K.; Kanesue, T. Nucl. Instrum. Methods A 2014, 733, 97-102.

(8) Boehne, D.; Hofmann, I.; Kessler, G.; Kulcinski, G.L.; Meyer-ter-Vehn, J.; von Moellendorff, U.; Moses, G.A.; Mueller, R.W.; Sviatoslavsky, I.N.; Sze, D.; Vogelsang, W. Nuclear Engineering and Design 1982, 73, 195-200.

(9) Badger, B.; Beckert, K; Bock, R.; Boehne, D.; Bozsik, I.; Brezina, J.; Corradini, M.; El-Guebaly, L.; Engelstad, R.; Froehlich, R. et al., University of Wisconsin report UWFDM-625 (1984), Kfk-3840 and FPA-84-4.

(10) Ikeda, S.; Kumaki, M.; Fuwa, Y.; Kanesue, T.; Okamura, M.; Horioka, K. Proceedings of International Particle Accelerator Conference 2014, Dresden, Germany, 2014, p. 601-603.

(11) Kanesue, T.; Okamura, M.; Alessi, J.; Beebe, E.; Pikin, A.; Raparia, D.; Liaw, C.J.; Lambiase, R.; LoDestro, V.; Costanzo, M. et al, Proceedings of International Particle Accelerator Conference 2014, Dresden, Germany, 2014, p. 1890-1892.

(12) Alessi, J.; Beebe, E.; Binello, S.; Gardner, C.; Gould, O.; Hoff, L.; Kling, N.; Lambiase, R.; Lockey, R.; LoDestro, V. et al., Proceedings of 2011 Particle Accelerator Conference, New York, USA, 2011, p. 1966-1968.

(13) Kanesue, T.; Tamura, J.; Okamura, M. Proceedings of European Particle Accelerator Conference 08, Genoa, Italy, 2008, p. 421-423.

(14) Munemoto, N.; Takayama, K.; Takano, S.; Okamura, M.; Kumaki, M. Rev. Sci. Instrum. 2014, 85, $02 \mathrm{~B} 922$. 


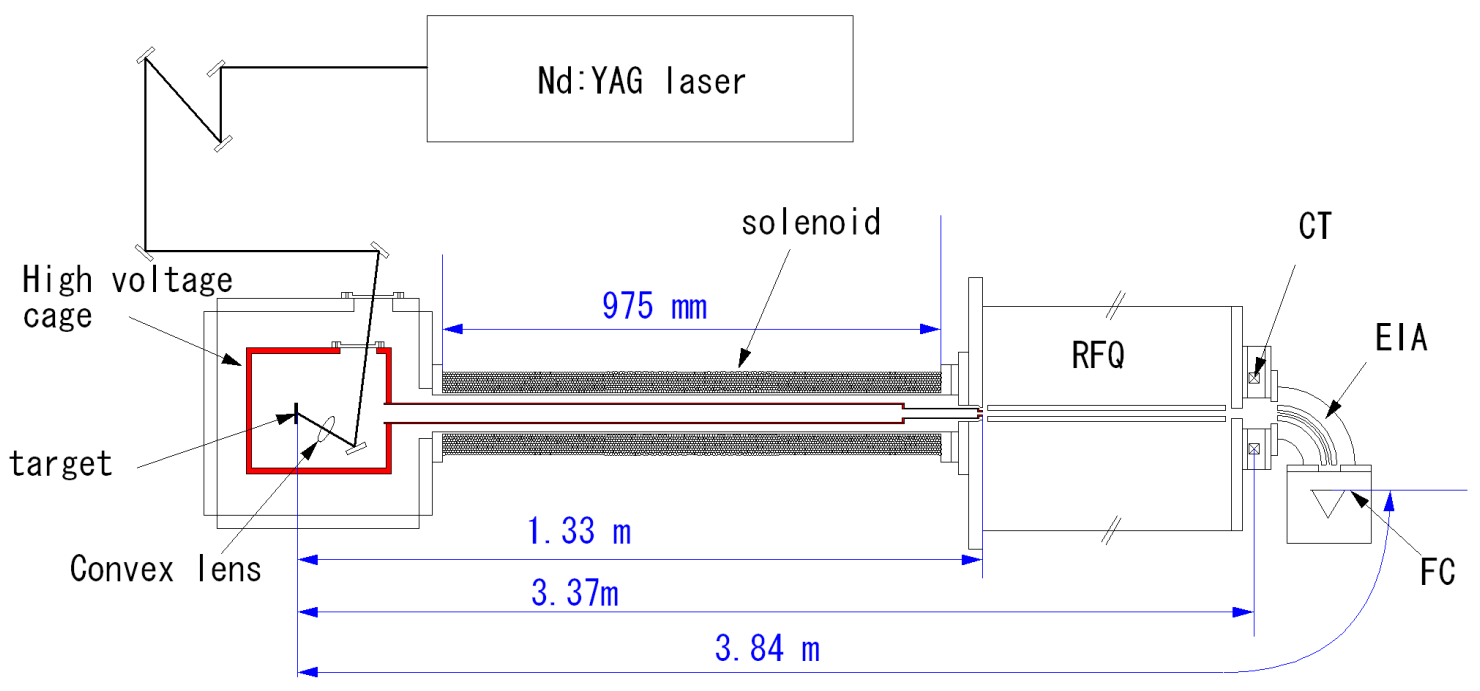

Figure 1 Apparatus of DPIS. 

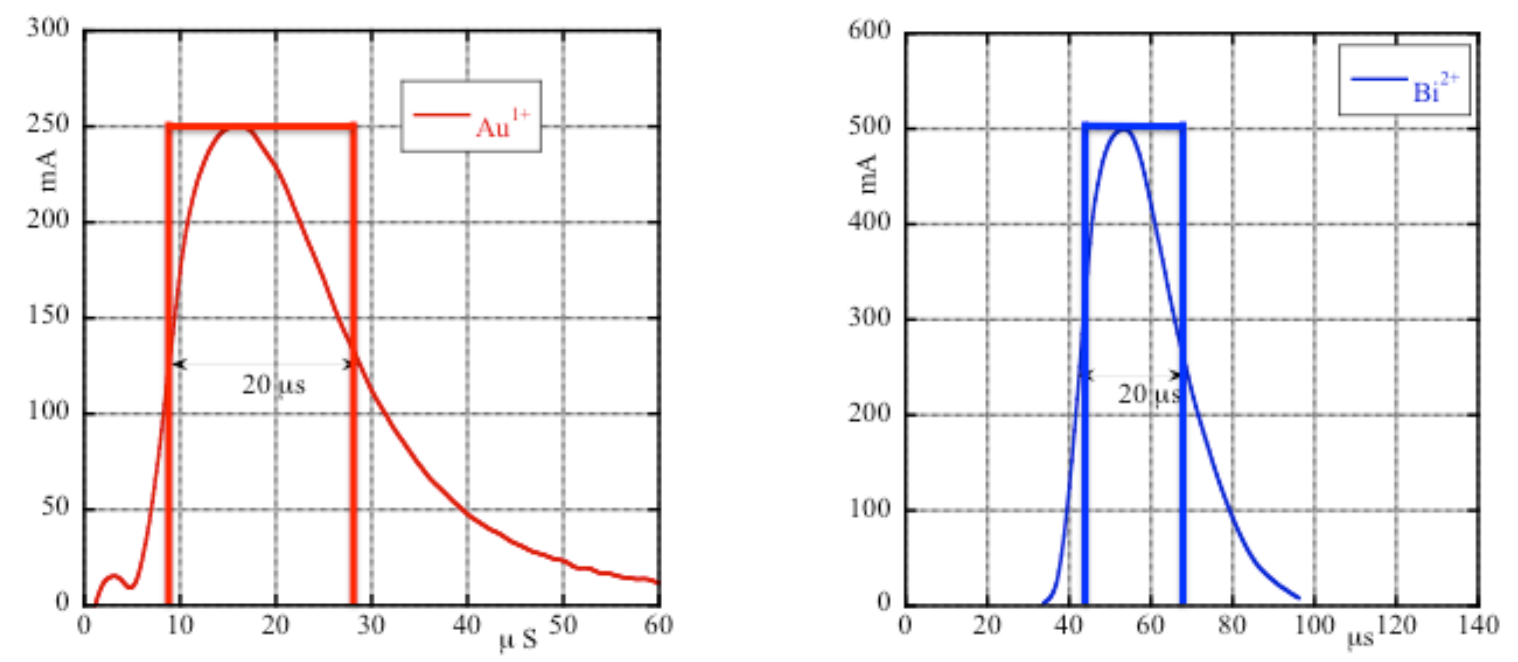

Figure 2. Expected current wave forms based on the measured results of $\mathrm{Au}$ and Bi productions. The square shapes show ideal current profiles. 


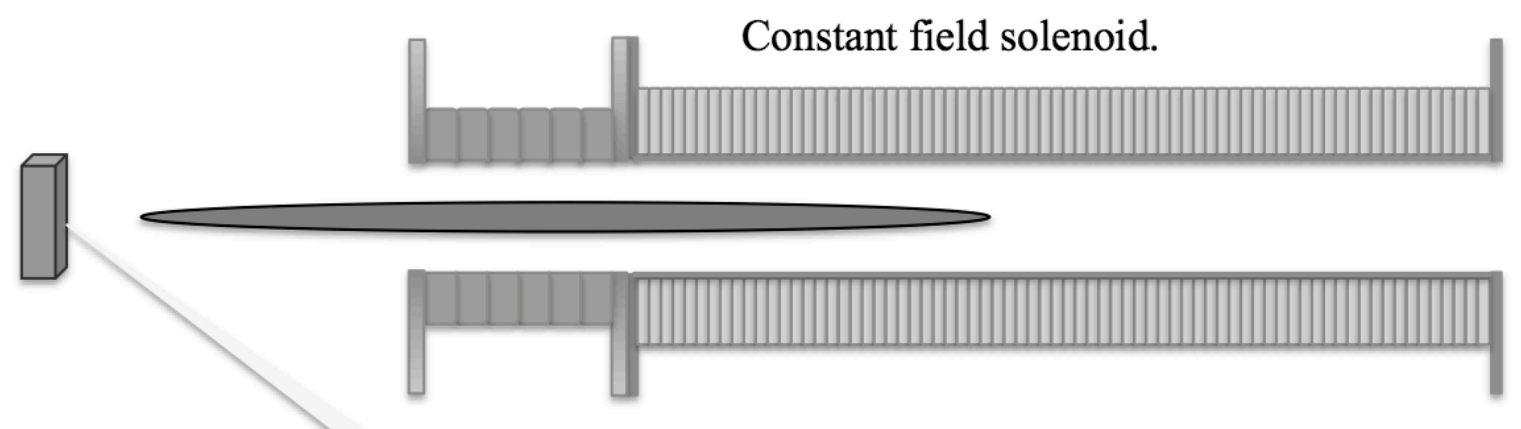

Pulsed solenoid.

Figure 3. Conceptual layout of pulsed solenoid. The rectangular target is irradiated by laser shot and the ablated plasma captured by the solenoids 


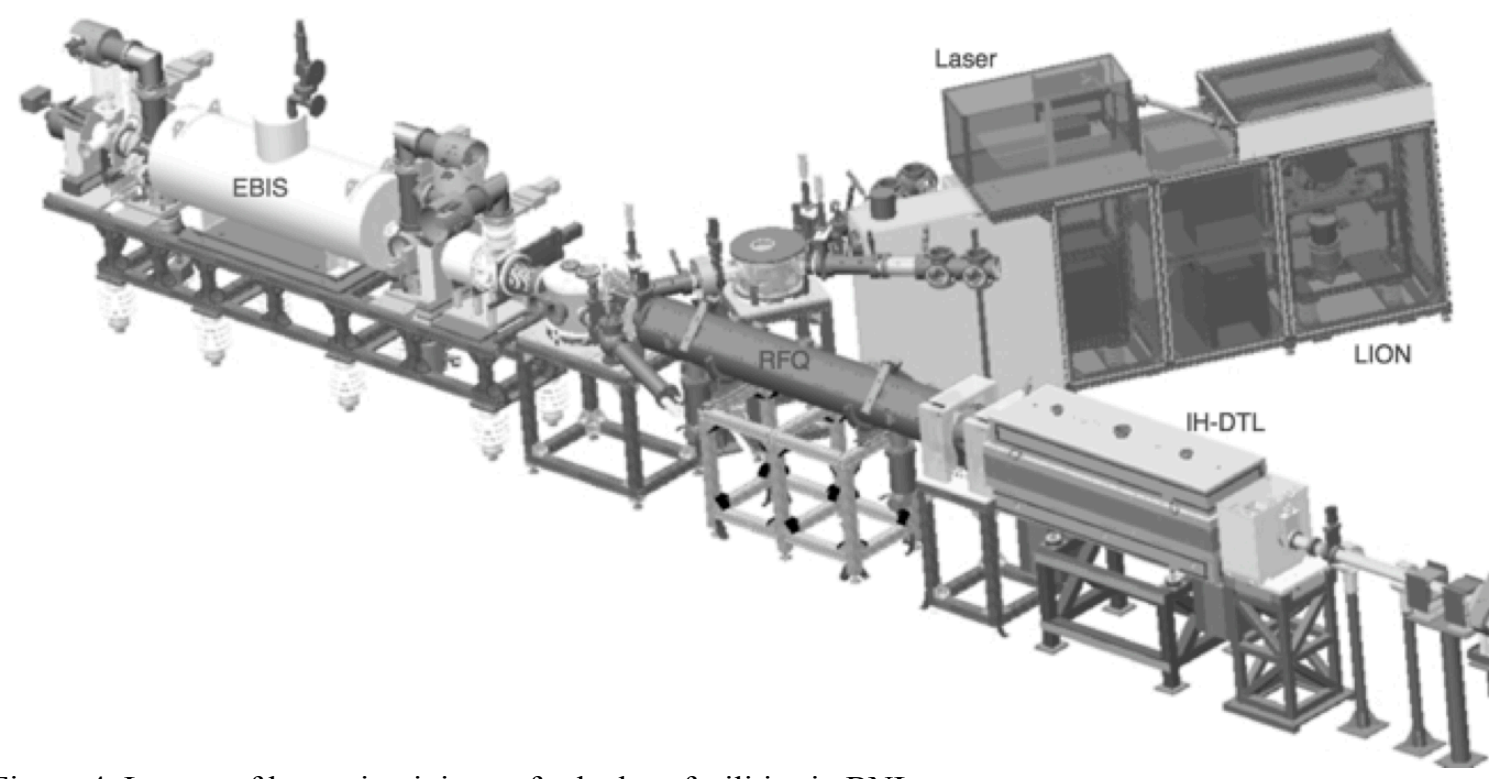

Figure 4. Layout of heavy ion injector for hadron facilities in BNL 


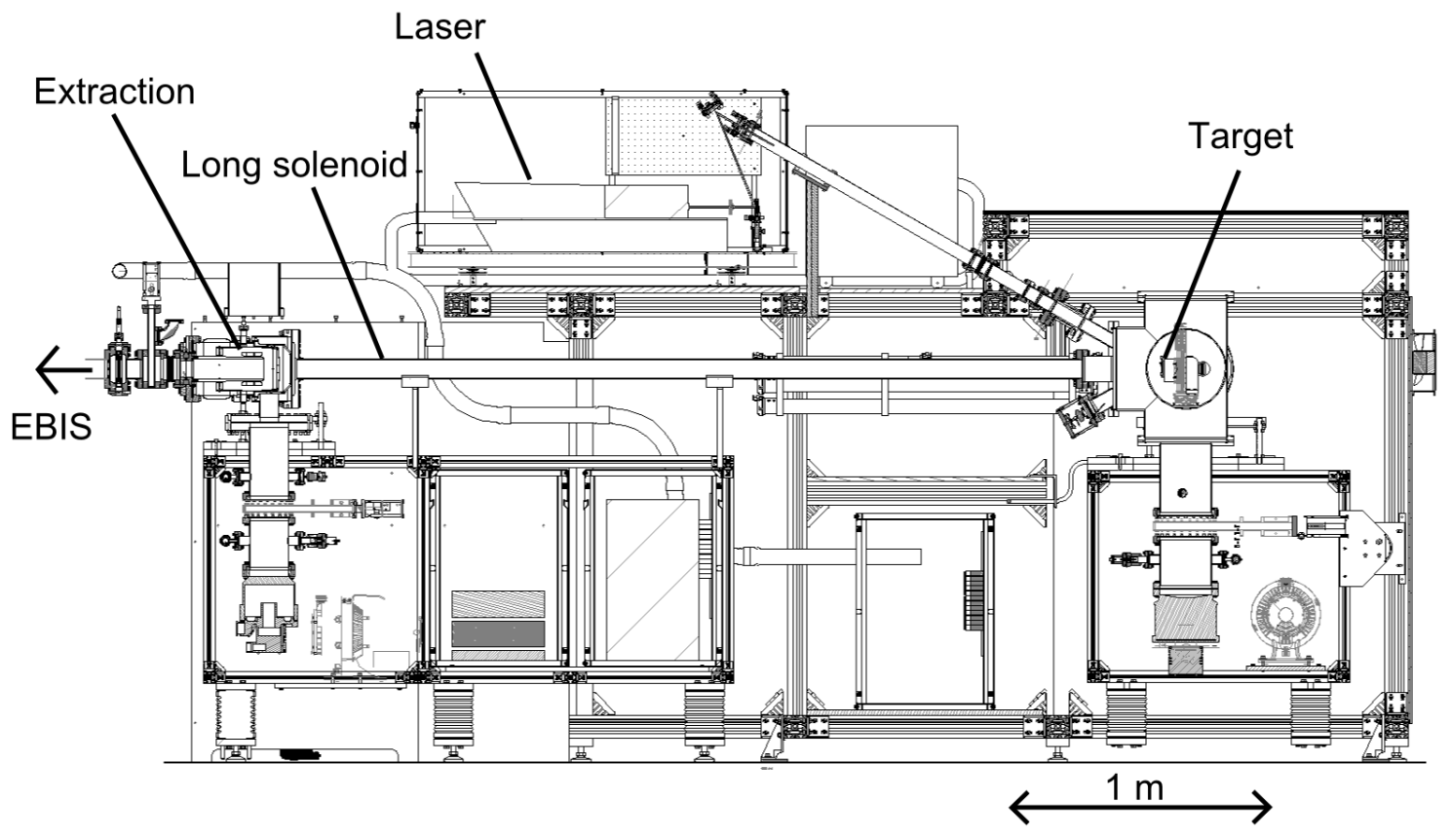

Figure 5. LION source 